\title{
An Improved Location Algorithm by Extend Square-root Cubature Kalman Filter
}

\author{
Ruiguo Sheng \\ Electronic Information Engineering Department of Naval Aeronautical and Astronautical \\ University, Yantai, China \\ Email: ruiguosheng@gmail.com \\ Yang Zhang and Jun Miao \\ Electronic Information Engineering Department of Naval Aeronautical and Astronautical \\ University, Yantai, China \\ Email: zhyang_111@163.com
}

\begin{abstract}
In this paper, the new nonlinear filter method Cubature Kalman Filter (CKF) is improved to solve the passive location problem. Firstly, the Empirical Mode Decomposition (EMD) algorithm is used to estimate the new measurement noise covariance in the filter process; And then the new covariance of the noise is brought into the circle; Meanwhile, the location process is improved by the way of square root to keep its stability and positivity, and the results of track with moving angle-measured sensors' measurements by Extend SCKF are compared with the results by Unscented Kalman Filter (UKF) in the paper; By the tracking results to the velocity of the target, Extend SCKF algorithm can not only track the target with unknown measurement noise but also improve the passive position precision remarkably with the same complexity of UKF algorithm.
\end{abstract}

Index Terms-Cubature Kalman Filter, Sensor, Empirical Mode Decomposition, Square-Root Cubature Kalman Filter, Unscented Kalman Filter

\section{INTRODUCTION}

With the development of the sensor technology, modern network and communication technology, multistation angle sensor passive positioning [1, 2, 3, 4] has been increasingly used because of its simple construction, positioning precision, and it plays an important role in reconnaissance, rescue, aerospace, aviation, marine and other fields.

In modern passive locations by sensors, the problem is often transformed into the additional noise filtering problem of nonlinear dynamic systems, filter model can be transformed into a discrete time differential equation [5], which is the state equation and measurement equation of the target.

With the further research of modern sensor passive location, it is found that tracking research often faces two

Manuscript received November 07, 2011

This work was supported by special funding of "Taishan Scholar" Construction in China. difficulties: 1 . How to improve the accuracy of the target tracking under a low computational com-plexity; 2. Recursive estimation problem under unknown noise filter prior information.

For the first question, in recent years, scholars have put forward a variety of algorithms, such as: Extend Kalman filter (EKF) algorithm [6] using the first order Taylor expansion.Unscented Kalman Filter (UKF) algorithm [7] based on the UT transform sampling (Sigma) points, Under a considerable complexity in the computation, UKF algorithm's accuracy is higher than EKF algorithm, it would become widely used in recent year as a nonlinear filtering algorithm. Recently, a new nonlinear filter-ing method-Cubature Kalman filter (CKF) algorithm [8-9] has been proposed, which uses a set of weights as the cubature point sets to solve the integration of Bayesian filtering problem for a nonlinear estimation problem. To ensure the algorithm's stability and positive definition, the paper uses a robust improvement of CKF form-SCKF as the main algorithm for passive locations.

In addition, in the process of locating and tracking by sensors, the noise of the tracking is often assumed by experience, which assumes a priori statistical information of targets noise, in practical engineering it is often difficult to achieve. Empirical mode decomposition (EMD) algorithm [10] is introduced to estimate the priori statistical information of noise on the target to meet the needs of the actual sensor passive location and tracking, while the process noise covariance and measurement noise covariance of the target are taken into the whole cycle, and further the algorithm accuracy is improved.

\section{A BAYESIAN FILTERING PROCESS}

Nature of tracking is a Bayesian filtering cycle [11], which is as follows in Figure 1 . The tracking may be transformed to the problems how to solve the posterior probability density of target state. To obtain the status of time $k$, the paper uses the following two basic steps to update the state posterior probability density of the time $(k-1)$. 


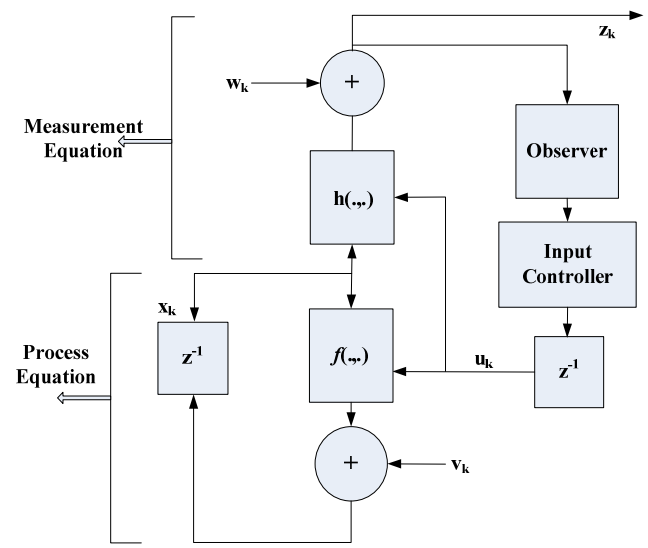

Fig. 1 Process of the cycle filter

A. Time Update, Which Calculates the Forecast Probability Density :

$$
p\left(x_{k} \mid D_{k-1}\right)=\int_{R^{n_{x}}} p\left(x_{k-1} \mid D_{k-1}\right) \times p\left(x_{k} \mid x_{k-1}, u_{k-1}\right) d x_{k-1}
$$

Here $D_{k-1}=\left\{u_{i}, z_{i}\right\}_{i=1}^{(k-1)}$ denotes the history values of the input measurement to the Moment $(k-1)$, $p\left(x_{k-1} \mid D_{k-1}\right)$ is the posterior probability density of time $(k-1)$,and the state transition probability density $p\left(x_{k} \mid x_{k-1}, u_{k-1}\right)$ can be obtained from (1).

B.Measurement Update, Which Calculates the Posterior p-robability Density of the Current State:

$$
p\left(x_{k} \mid D_{k}\right)=p\left(x_{k} \mid D_{k-1}, u_{k}, z_{k}\right)
$$

Using Bayesian norms:

$$
p\left(x_{k} \mid D_{k}\right)=\frac{1}{c_{k}} p\left(x_{k} \mid D_{k-1}, u_{k}\right) p\left(z_{k} \mid x_{k}, u_{k}\right)
$$

$c_{k}$ is a constant state obtained by the following formula:

$$
\begin{aligned}
& c_{k}=p\left(z_{k} \mid D_{k-1}, u_{k}\right)= \\
& \int_{R^{n_{x}}} p\left(x_{k} \mid D_{k-1}, u_{k}\right) p\left(z_{k} \mid x_{k}, u_{k}\right) d x_{k}
\end{aligned}
$$

According to the relation of the prior and posterior probability of (2), the input needs to satisfy the following relationship:

$$
p\left(u_{k} \mid D_{k-1}, x_{k}\right)=p\left(u_{k} \mid D_{k-1}\right)
$$

There should be enough information of input $u_{k}$ to be produced by the control $D_{k-1}$. Specifically, the input $u_{k}$ is present by $\hat{x}_{k \mid k-1}$. In this case, it can be written equivalently as (6),

$$
p\left(x_{k} \mid D_{k-1}, u_{k}\right)=p\left(x_{k} \mid D_{k-1}\right)
$$

Therefore, we substitute (3) into (2), get (7),

$$
p\left(x_{k} \mid D_{k}\right)=\frac{1}{c_{k}} p\left(x_{k} \mid D_{k-1}\right) p\left(z_{k} \mid x_{k}, u_{k}\right)
$$

Under the assumption,

$$
c_{k}=\int_{R^{n_{x}}} p\left(x_{k} \mid D_{k-1}\right) p\left(z_{k} \mid x_{k}, u_{k}\right) d x_{k}
$$

But in reality, when faced with a nonlinear filtering problem, it's difficult to obtain the optimal solution of the Bayesian posterior probability density; we need to seek the suboptimal solution of the Bayesian filtering. In calculation process, posterior probability density for the suboptimal solution can be obtained by the following two methods:

Global method:

The Sampling methods for Bayesian recursion are used. For example, Gaussian mixture filter method, particle filter[12] which uses importance sampling Monte Carlo calculation, etc., all belong to this kind, but these global approach requires a great computation complexity. Local method:

Nonlinear filter is achieved by changes of posterior probability density. For example, Gaussian nonlinear filtering methods, such as EKF (Extended Kalman filter), CDKF [13] (Central Difference Kalman Filter), UKF (Unscented Kalman Filter), are of this type. The emphasis on local information makes the filtering process simple and quick.

\section{COMPARISON OF TWO NONLINEAR FILTERING ALGORITHMS}

\section{A. UKF (Unscented Kalman Filter) Algorithm}

As a representative local methods, Unscented Kalman Filter (UKF) is a Bayesian filter which is based on the Gaussian field approximation, which is characterized by using a method known as the UT transformation to the recursive operation, considering a random variable with mean $\mu$ and covariance $\Sigma$, which needs $(2 n+1)$ sampling points, and the weights $\left\{\chi_{i}, \omega_{i}\right\}_{i=0}^{2 n}$ meet:

$$
\begin{gathered}
\sum_{i=0}^{2 n} \omega_{i} \chi_{i}=\mu \\
\sum_{i=0}^{2 n} \omega_{i}\left(\chi_{i}-\mu_{x}\right)\left(\chi_{i}-\mu_{x}\right)^{T}=\Sigma
\end{gathered}
$$

Here, series of distributed point sets known as the sigma point set, can be described as follows:

$$
\begin{gathered}
\chi_{0}=\mu, \omega_{0}=\frac{\kappa}{n+\kappa} \\
\chi_{i}=\mu+(\sqrt{(n+\kappa) \Sigma})_{i}, \omega_{i}=\frac{1}{2(n+\kappa)} \\
\chi_{n+i}=\mu-(\sqrt{(n+\kappa) \Sigma})_{i}, \omega_{n+i}=\frac{1}{2(n+\kappa)}
\end{gathered}
$$

Parameters $\kappa$ is used as the sigma point from the Priori mean $\mu$, which is called scale parameter. In order to obtain the peak of the priori probability density, generally choose $\kappa$ as $\kappa=(3-n)$.

In summary, the sigma-point sets are chosen to capture a number of low-order moments of the prior density $p(x)$ as correctly as possible.

Then the unscented transformation is introduced as a method of computing posterior statistics that are related to $x$ by a nonlinear transformation $y=f(x)$. It approximates the mean and the covariance of $y$ by a weighted sum of projected sigma points in the real space, as shown by 


$$
\begin{gathered}
E[y]=\int_{R^{n}} f(x) \Pi(x) d x \approx \sum_{i=0}^{2 n} \omega_{i} y_{i} \\
\operatorname{cov}[y]=\int_{R^{n}}(f(x)-E[y])(f(x)-E[y])^{T} \Pi(x) d x \\
\approx \sum_{i=0}^{2 n} \omega_{i}\left(y_{i}-E[y]\right)\left(y_{i}-E[y]\right)^{T}
\end{gathered}
$$

Because the method of passive location sensor is widely used, it is necessary to achieve fast positioning on the target, and try to avoid the reduction of accuracy, which urgently needs to find a better method of local filtering to passive sensors for more precise position-ing; Today the UKF Technology has matured, so it is gradually shifted to pursuit better filtering methods for tracking accuracy. Recently the new method of Kalman filter called CKF (Cubature Kalman filter) is proposed as a new filtering method, which provides a new way for solution.

\section{B. Comparison between UKF and CKF Algorithm \\ Common features:}

The UKF and the CKF share a common property- they use weighted sets of symmetric points.

Their points and weights are denoted by the location and the height of the stems, respectively.

The advantages of CKF:

Numerical accuracy. Traditionally, there has been more emphasis on cubature rules of the CKF algorithm which has desirable numerical quality criterion than on the efficiency.

Availability of a square-root solution. We perform the square-rooting operation (or the Cholesky factorization) on the error covariance matrix as the first step of both the time and measurement updates in each cycle of both the UKF and the CKF. From the implementation point of view, the square-root process is one of the most numerically sensitive operations. To avoid this operation in a systematic manner, we may seek to develop a squareroot version of the UKF. Unfortunately, it may be impossible for us to formulate the square-root UKF that enjoys numerical advantages similar to the Square-root Cubature Kalman Filter. The reason is that when a negatively weighted sigma point is used to update any matrix, the resulting down-dated matrix may possibly be non-positive definite. Hence, errors may occur when executing a pseudo square-root version of the UKF in a limited system.

Filter stability. Given no computational errors due to an arithmetic imprecision, the presence of the negative weight may still prohibit us from writing a covariance matrix $P$ in a squared form such that $P=S S^{T}$. To state it in another way, the UKF-computed covariance matrix is not always guaranteed to be positive definite.

Hence, the unavailability of the square-root covariance causes the UKF to halt its operation. This could be disastrous in practical terms. To improve stability, CKF can effectively avoid the disaster.

\section{EMD Algorithm}

Empirical mode decomposition method $^{[14-16]}$ is essentially a smooth processing for a signal (or its derivative, depending on the required accuracy of the decomposition), the result is that the different scales of fluctuations of the signal are decomposed, and series of sequences with different characteristic scales are produced, each sequence is called an intrinsic mode functions (Intrinsic Mode Function, IMF). The IMF component of the lowest frequency usually represents the tendency of the original signal. As an application, EMD decomposition method can effectively extract the tendency of the data sequences or remove the mean of the data sequences. Test results show that, EMD method is the best way [16] to extract the tendency or mean of the signal, EMD method is also aimed to achieve the Hilbert transform of each IMF component to obtain the instantaneous frequency of the signal.

By the smooth processing to the signal, it can be decomposed $n$ IMF components $c_{i}(t)$ and the tendency $r_{n}(t)$, namely

$$
x(t)=\sum_{i=1}^{n} c_{i}(t)+r_{n}(t)
$$

Each IMF component is the smooth signal.

The basic idea of EMD decomposition method (smoothing process) is: Find all the maximum points of the original data series and interpolate it as an upper envelope of the original data series with a spline interpolation function; and then find all the minimum points of the original data series and interpolate them as an lower envelope of the original data series with a spline interpolation function; the mean of the upper and lower envelope is the average envelope of the original data sequence, a new data series without high-frequency data can be got by the original data sequence after subtracting it to the average envelope .

In the frequency domain, instantaneous frequencies among each IMF of the signal by the EMD decomposition have the following relationship: The first IMF has the maximum instantaneous frequency component, and the $i$-th (of which $i \geq 2$ ) instantaneous frequency of the IMF is almost twice of the $(i+1)$-th instantaneous frequency of the IMF [16]. Therefore, each IMF of the signal decomposed by EMD can be seen as the band-pass filtering results of the original signal [1618]. EMD decomposition algorithm can be achieved through spatial and temporal scale filter, whose low-pass filter can be denoted as (17):

$$
X(t)=\sum_{j=k}^{n} I M F_{j}+r(t)
$$

As the frequency of the noise is generally higher than the signal, while the frequency of each IMF is reduced almost in the form [17] of a negative power of 2, so all the noise contained in the IMF will become more and more weak, and the low frequency part of IMF is almost the low-frequency component of the expected signal.

\section{Extend SCKF Algorithm}

UKF algorithm may usually face a problem which is how to maintain the positive definition of the error 
covariance, the recursive filter by the CKF algorithm may also meet the problem, the paper improves the CKF algorithm by the form of the square root, not only can further improve the algorithm's accuracy, but effectively ensure that the process of the algorithm are positive defined.

EMD (EMD) method can separate signal and noise, so it can be used to estimate the standard deviation of the noise in the signal. In the case that observation noise is unknown; we can first select a section of signal with length $L$, which is equivalent in an observation interval. The empirical mode decomposition is used in the observation interval, separating the noise of the high frequency out, and then the observation noise Standard deviation can be estimated.

In addition, the paper also brings the new covariance of the noise into the entire cycle, and further improves the accuracy of the algorithm.

Figure 2 shows the entire process of the recursive filter by the algorithms.

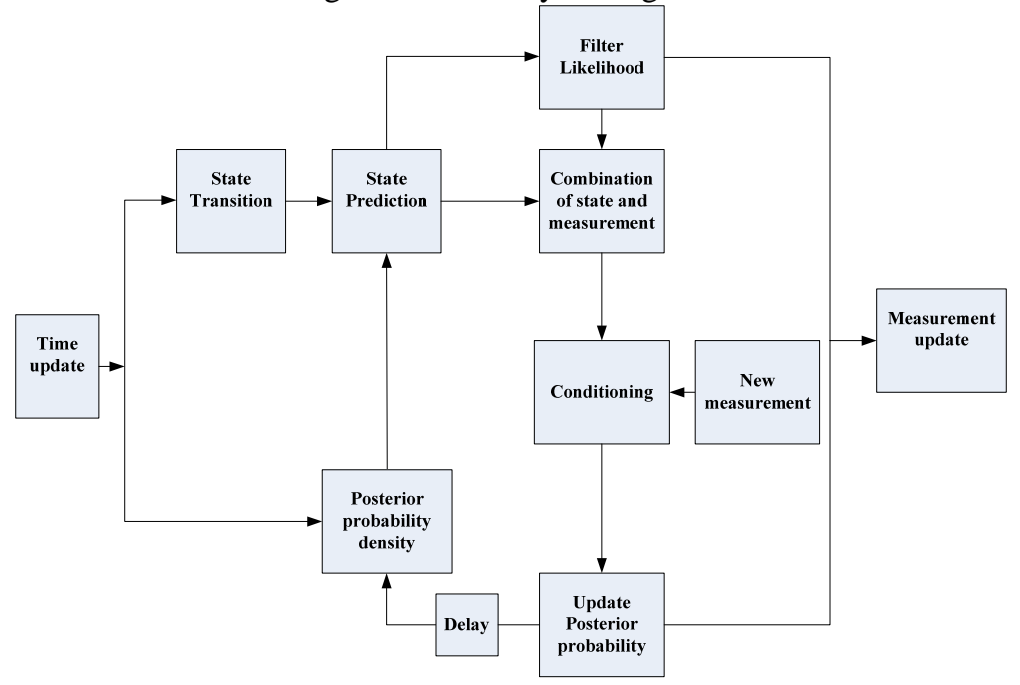

Fig. 2. Signal-flow diagram of the recursive filter

\section{E. Time Update}

Assume the posterior probability density function at the moment $k-1: p\left(x_{k-1} \mid D_{k-1}\right)=N\left(x_{k-1 \mid k-1}, P_{k-1 \mid k-1}\right)$ is known. Decomposed:

$$
P_{k-1 \mid k-1}=S_{k-1 \mid k-1} S_{k-1 \mid k-1}^{T}
$$

Estimate the cubature points $(i=1,2, \ldots, m)$ :

$$
X_{i, k-1 \mid k-1}=S_{k-1 \mid k-1} \xi_{i}+x_{k-1 \mid k-1}
$$

Here, $m=2 n_{x}$,

$$
\xi_{i}=\sqrt{n_{x}}\left\{\left(\begin{array}{c}
1 \\
\ldots \\
0
\end{array}\right),\left(\begin{array}{c}
0 \\
\ldots \\
1
\end{array}\right), \cdots,\left(\begin{array}{c}
-1 \\
\ldots \\
0
\end{array}\right),\left(\begin{array}{c}
0 \\
\ldots \\
-1
\end{array}\right)\right\}
$$

Estimate the propagated cubature points

$$
X_{i, k \mid k-1}^{*}=f\left(X_{i, k-1 \mid k-1}, u_{k-1}\right)
$$

Estimate the predictive state

$$
x_{k \mid k-1}=\frac{1}{m} \sum_{i=1}^{m} X_{i, k \mid k-1}^{*}
$$

Estimate the square root of the predictive error covariance

$$
S_{k \mid k-1}=\operatorname{Tria}\left(\left[\chi_{k \mid k-1}^{*} \quad S_{Q, k-1}\right]\right)
$$

Tria(·) represents the low triangular matrix by $\mathrm{QR}$ decomposition, here $S_{Q, k-1}$ represents the square root factor of $Q_{k-1}$, here $Q_{k-1}=S_{Q, k-1} S_{Q, k-1}^{T}$ and the weight, the center matrix (a priori mean is subtracted):

$$
\chi_{k \mid k-1}^{*}=\frac{1}{\sqrt{m}}\left[X_{1, k \mid k-1}^{*}-x_{k \mid k-1} \quad \cdots \quad X_{m, k \mid k-1}^{*}-x_{k \mid k-1}\right]
$$

\section{F. Measurement Update}

Estimate the cubature points $(i=1,2, \ldots, m)$

$$
X_{i, k \mid k-1}=S_{k \mid k-1} \xi_{i}+x_{k \mid k-1}
$$

Estimate the propagated cubature points

$$
Z_{i, k \mid k-1}=h\left(X_{i, k \mid k-1}, u_{k}\right)
$$

Make the EMD estimation to the measurement noise, follow the steps:

(1)Calculate all the extreme points of $Z_{i, k \mid k-1}$.

(2)Use the cubic spline interpolation algorithm [13], and solve the upper envelope composed by all the maxima and the lower envelope of all the minimum points, denoted by $u_{1}(t)$ and $v_{1}(t)$;Meanwhile extend the extreme points to suppress edge effects by mirror extension approach [14, 15].

(3)Record the mean of the upper envelope and the lower envelope:

$$
m_{1}(t)=\frac{u_{1}(t)+v_{1}(t)}{2}
$$

Record the difference between the envelope mean and the signal:

$$
h_{1}^{(1)}(t)=z(t)-m_{1}(t)
$$


(4)Judge whether $h_{1}^{(1)}(t)$ satisfies the two properties of the IMF (Intrinsic Mode Function). If satisfied, then $h_{1}^{(1)}(t)$ is IMF.Otherwise, record $h_{1}^{(1)}(t)$ is $Z_{i, k \mid k-1}$, repeat step one to step four, assume $h_{1}^{(1)}(t)$ selected $k$ times satisfies the IMF definition, and the first order IMF of $Z_{i, k \mid k-1}$ is $c_{1}(t)=h_{1}^{(1)}(t)$.

(5)Record $Z_{1}(t)=Z_{i, k \mid k-1}-c_{1}(t)$ as the new signal to be analyzed. Repeat the step (1) to the step (5) to obtain the second IMF, and the remaining items $Z_{2}(t)=Z_{1}(t)-c_{2}(t)$ Repeat the above steps until the remaining item is a monotone signal. Finally, the remaining item $Z_{m}(t)$ can be obtained, $Z_{m}(t)$ is the observation of measurement noise, and $R_{k}$ is determined.

Measurement estimation

$$
z_{k \mid k-1}=\frac{1}{m} \sum_{i=1}^{m} Z_{i, k \mid k-1}
$$

Estimate the square root of the update covariance matrix

$$
S_{z z, k \mid k-1}=\operatorname{Tria}\left(\left[\begin{array}{ll}
\Gamma_{k \mid k-1} & S_{R, k}
\end{array}\right]\right)
$$

Here $S_{R, k}$ denotes a square root factor of $R_{k}$, and $R_{k}=S_{R, k} S_{R, k}^{T}$, and its weight of the center matrix is:

$$
\Gamma_{k \mid k-1}=\frac{1}{\sqrt{m}}\left[Z_{1, k \mid k-1}-Z_{k \mid k-1} \quad \cdots \quad Z_{m, k \mid k-1}-Z_{k \mid k-1}\right]
$$

Estimate the cross-covariance matrix

$$
P_{x z, k \mid k-1}=\chi_{k \mid k-1} \Gamma_{k \mid k-1}^{T}
$$

The weight of the center matrix here is:

$$
\chi_{k \mid k-1}=\frac{1}{\sqrt{m}}\left[X_{1, k \mid k-1}-x_{k \mid k-1} \quad \cdots \quad X_{m, k \mid k-1}-x_{k \mid k-1}\right]
$$

Obtain the Kalman gain

$$
K=\left(P_{x z, k \mid k-1} / S_{z z, k \mid k-1}^{T}\right) / S_{z z, k \mid k-1}
$$

Update the state estimation

$$
x_{k \mid k}=x_{k \mid k-1}+K\left(z_{k}-z_{k \mid k-1}\right)
$$

Estimate the square root of error covariance

$$
S_{k \mid k}=\operatorname{Tria}\left(\left[\chi_{k \mid k-1}-K \Gamma_{k \mid k-1} \quad K S_{R, k}\right]\right)
$$

Further, $P_{k \mid k}=S_{k \mid k} S_{k \mid k}^{T}$.

Take the process noise covariance and measurement noise covariance into the recycling process

The new covariance matrix is $P_{k \mid k}^{*}=\left(\begin{array}{ccc}P_{k \mid k} & 0 & 0 \\ 0 & Q_{k} & 0 \\ 0 & 0 & R_{k}\end{array}\right)$, resubstituted it into the loop, and (18), (25) are changed to

$$
\begin{gathered}
S_{k \mid k-1}=\operatorname{Tria}\left(\chi_{k \mid k-1}^{*}\right) \\
S_{z z, k \mid k-1}=\operatorname{Tria}\left(\Gamma_{k \mid k-1}\right)
\end{gathered}
$$

\section{Simulation TESTS}

\section{A. Simulation Conditions}

The experimental coordinate is assumed the Cartesian coordinate, the total test time is $800 \mathrm{~s}$, experiments times is five, the target set in the initial position is $(120,100)$, state noise is set to white noise with mean 0 and variance 0.01 ,the initial position of three sensors, are respectively $(-100,0),(0,0),(100,0)$. Set the measurement noise Gaussian white noise, whose mean is 0 and variance is set randomly in the interval $\left[10^{-6}, 10^{-5}\right],\left[10^{-5}, 10^{-4}\right],\left[10^{-4}, 10^{-3}\right]$.

Assume that the initial system error covariance matrix is

$$
P_{0}=\left[\begin{array}{cccc}
\sigma^{2} & \sigma^{2} / T & 0 & 0 \\
\sigma^{2} / T & 2 \sigma^{2} / T^{2} & 0 & 0 \\
0 & 0 & \sigma^{2} & \sigma^{2} / T \\
0 & 0 & \sigma^{2} / T & 2 \sigma^{2} / T^{2}
\end{array}\right]
$$

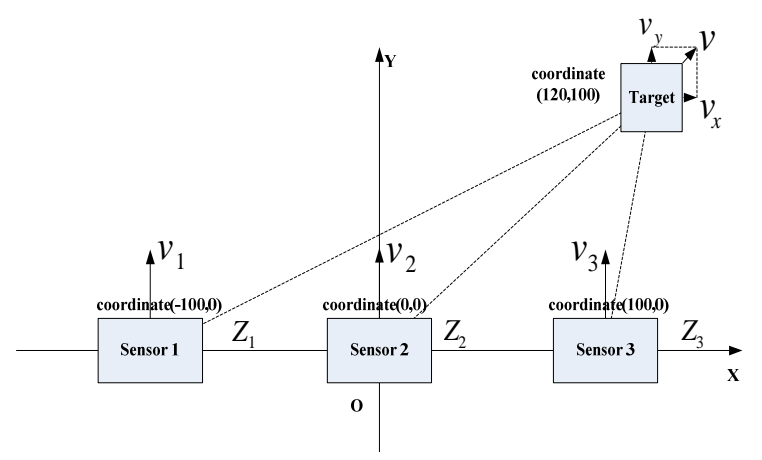

Fig.3 Target tracking figure by the angles of three sensors

Specific simulation scenario are shown as Figure 3, the movement of the target and the sensor were made two assumptions:

(1)Pure angle sensor tracking ,target and sensor makes uniform movement, the speed of the goal in $\mathrm{x}$, $\mathrm{y}$ direction are all $0.3 \mathrm{~m} / \mathrm{s}$, the speed of three sensors are all $3 \mathrm{~m} / \mathrm{s}$;

(2) Pure angle sensor tracking,target and sensor makes uniform movement, the speed of the goal in $\mathrm{x}$, $\mathrm{y}$ direction are all $3 \mathrm{~m} / \mathrm{s}$, the speed of three sensors are all $30 \mathrm{~m} / \mathrm{s}$.

\section{B. Simulation Results}

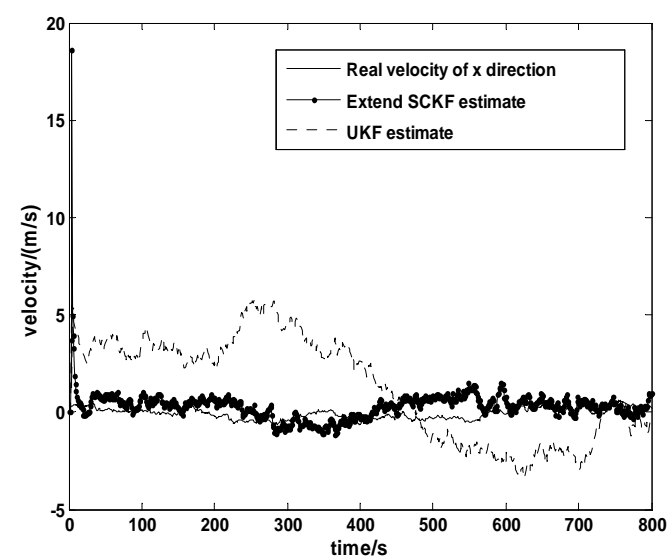

Fig. 4 The first velocity tracking figure 
Here only shows the target tracking results of the $\mathrm{x}$ direction, as shown below:

Figures 4, Figure 5 represent the comparison of target speed tracking and mean square error according to the assumption (1);

Figures 6, Figure 7 represent the comparison of target speed tracking and mean square error according to the assumption (2);

Relative Velocity Error ( $R V E$ ) is defined to describe the performance of the filter, just as (39),

$$
R V E=\frac{\sqrt{\left(v_{\text {xtrue }}-\hat{v}_{x}\right)^{2}+\left(v_{\text {ytrue }}-\hat{v}_{y}\right)^{2}}}{\sqrt{v_{\text {xtrue }}{ }^{2}+v_{\text {ytrue }}{ }^{2}}}
$$

Where $\left(v_{x t r u e}, v_{y t r u e}\right)$ is the true velocity value, and $\left(\hat{v}_{x}, \hat{v}_{y}\right)$ is the estimated velocity value.

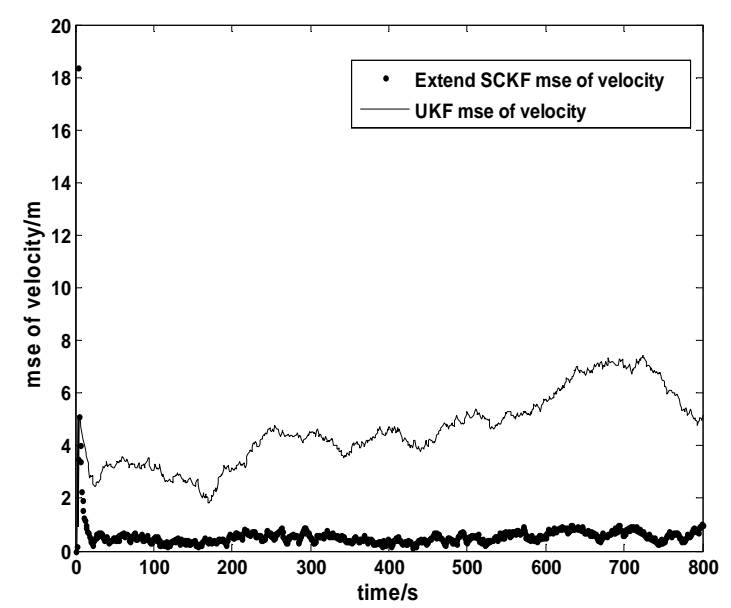

Fig. 5 MSE of first velocity tracking

Figure 4, Figure 5 shows, UKF and Extend SCKF all can track the speed of the target in $x$ direction, but the Extend SCKF is better than UKF on tracking accuracy obviously.

When the target speed and the sensor speed increase, target tracking accuracy is greatly affected.

The RVE comparison between the Extend-SCKF algorithm and the CKF algorithm is shown as Table 1; it is obvious that the filtering accuracy of Extend-SCKF algorithm is higher than that of CKF which uses the fixed variance by experience.

TABLE 1

COMPARISON OF THE AVERAGE RELATIVE ERROR BETWEEN EXTENDSCKF ALGORITHM AND CKF ALGORITHM

\begin{tabular}{|c|c|c|}
\hline $\begin{array}{c}\text { Variance of measurement } \\
\text { noise }\end{array}$ & Extend-SCKF & CKF \\
\hline$\left[10^{-6}, 10^{-5}\right]$ & $0.63 \%$ & $1.28 \%$ \\
\hline$\left[10^{-5}, 10^{-4}\right]$ & $0.85 \%$ & $1.49 \%$ \\
\hline$\left[10^{-4}, 10^{-3}\right]$ & $1.19 \%$ & $2.23 \%$ \\
\hline
\end{tabular}

The tracking results are shown as Figure 6 and Figure 7, it shows that even if the target tracking by UKF filter is divergent, the Extend SCKF algorithm can still achieve a stable target tracking.

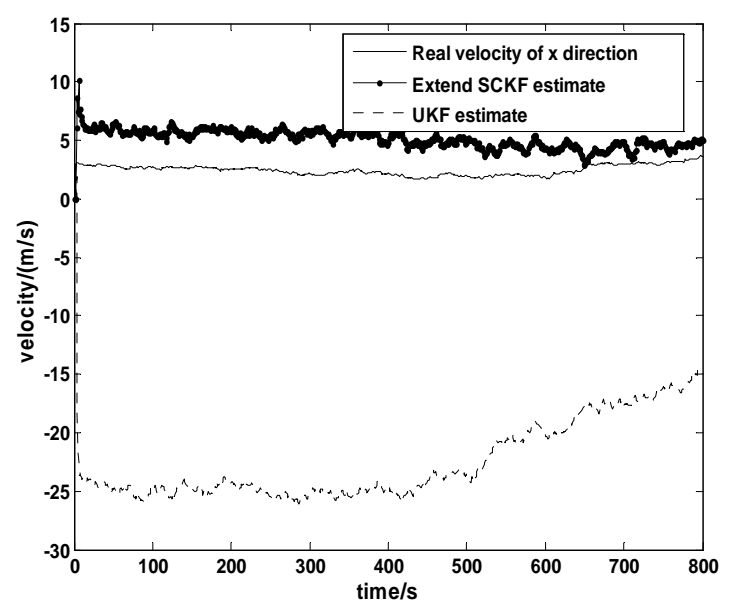

Fig. 6 The second velocity tracking figure

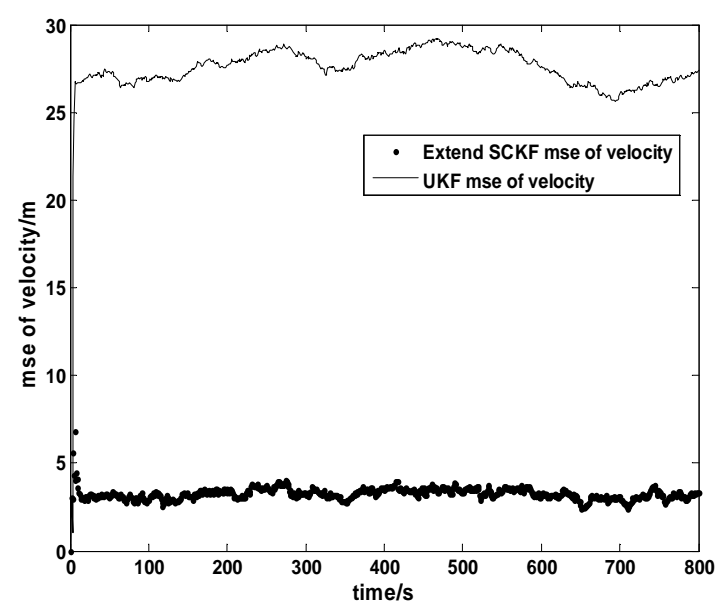

Fig. 7 MSE of second velocity tracking

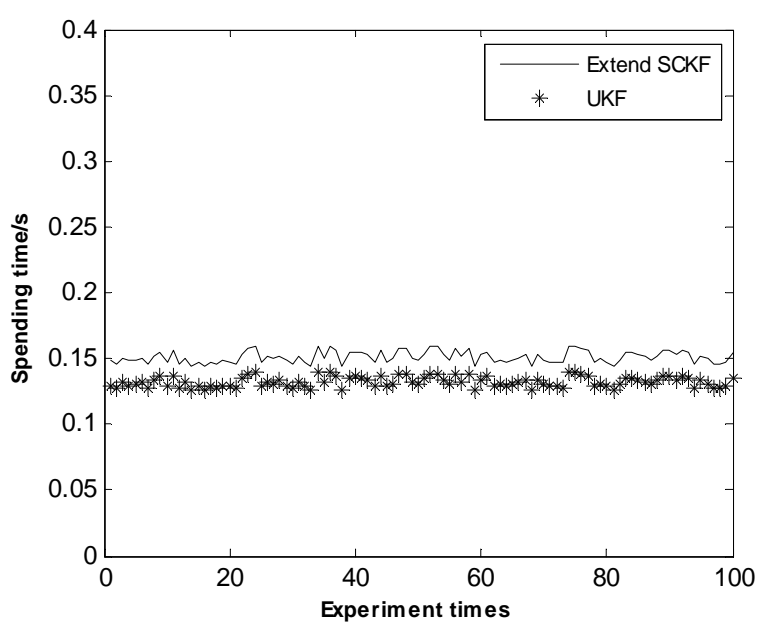

Fig. 8 Comparison of computation time

The time consumed by Extend SCKF algorithm is mainly concentrated in the EMD estimation of the measurement noise statistics. As shown in Figure 8, it is the comparison of computational complexity about the two algorithms, which is the time comparison of the target location by 100 times Monte Carlo simulations, it can be seen that Extend SCKF algorithm's computational complexity is equivalent to UKF algorithm. 


\section{CONCLUSION}

In the multi-sensor passive positioning problems, the research of the recursive tracking algorithm is always a hot spot, whose essence is the process to solve the Bayesian posterior probability density problem. The paper takes the target tracking with three moving anglesensors as the background, Extend SCKF algorithm which improves a new nonlinear filtering algorithm CKF is proposed for the recursive tracking of the target speed. Simulation results show that when the speed of the sensor and the target choose different values, compared with the traditional UKF algorithm, Extend SCKF algorithm can not only track the target when the measurement noise is unknown, but also significantly improves the tracking accuracy with the same computational complexity.

\section{REFERENCES}

[1] Sun Zhong Kang. Active and Passive Location technology With Single or Multiple Base [M]. Beijing: National Defense Industry Press, 1996: 42-46.

[2] Quan Tai Fan. New theory and technology of tracking [M]. Beijing: National Defense Industry Press, 2009, 50 64.

[3] Wu Ling.UKF algorithm and its applications to passive target tracking [J]. Systems Engineering and Electronics, 2005, 27 (1): 49-51.

[4] Kang Jian, Si Xi Cai. Nonlinear Filtering Techniques of Passive Location and Tracking. Systems Engineering and Electronics, 2004, 26 (2) : 161 162.

[5] Liu Mei, Quan Tai Fan, Yao Tian BIN. Multi-sensor multi-target tracking algorithm for passive location [J].Chinese Journal of Electronics. 2006, 34 (6): 991-995.

[6] Liu Qing Fu, Lu Yan E. Precision Analysis and Positioning Algorithm for Triple-star Passive Positioning [J]. Acta Aeronautical et Astronautic Sinica, 2008, 29(2): 456 461.

[7] I. Arasaratnam, S. Haykin, and R. J. Elliot. Discrete time nonlinear filtering algorithms using Gauss-Hermit quadrature. Proceedings of the IEEE, 95(5): 953-977, May 2007. 27(1): 49-51.

[8] S. Julier, J. Uhlmann, and H. F. Durrant-Whyte.A new method for the nonlinear transformation of means and covariances in filters and estimators. IEEE Transactions on Automatic Control, 45(3): 477-482, March 2000.

[9] Ienkaran Arasaratnam, Simon Haykin, Cubature Kalman Filtering for Continuous-Discrete Systems: Theory and Simulations. IEEE Transactions on Signal Processing, 58(10): 4977-4993, October 2010.
[10] Du Chen Yan, Zhang Yu Feng, Yang Ping. EMD edge effect suppressed Methods [J]. Chinese Journal of Science Instrument, 2009, 30 (1): 55-59.

[11] Gong Xiao Lin, Fang Jian Cheng. Application of Model Predictive Filtering Method in POS for Airborne SAR Motion Compensation System [J]. Acta Aeronautical et Astronautic Sinica, 2008, 29(1):102 109.

[12] Zhang Xing Ru. Performance Analysis of Passive Location and Location [D]. Chengdu University of Electronic Science and Technology, 2001. 02: 13-35.

[13] Zhong Dan Xing. Estimation of Rate of DOA Changes and Location of LBI Structured Passive Location System Boarded on a Moving Observer with Vibrate Attitudes [J]. Acta Aeronautical et Astronautic Sinica, 2007, 28(4):907 912.

[14] Liu Hui Ting, Hang Min, Cheng Jia Xing. Dealing with the End Issue of EMD Based on Polynomial Fitting Algorithm [J]. Computer Engineering and Applications, 2004, 40 (16):84-86.

[15] Yan Shi Xi, Hu Jin Song, Wu Zhao Tong. The Comparison of Vibration Signals' Time-Frequency Analysis Between EMD-Based HT and WT Method in Rotating Machinery [J]. Proceedings of the CSEE, 2003, 23(6): 119-121.

[16] Tan Shan Wen, Qin Shu Ren, and Tang Bao Ping. The Filtering Character of Hilbert-Huang Transform and Its Application [J]. Journal of Chong Qing University, 2004, 27(2) : 9-12.

[17] Deng Yong Jun, Wang Wei, Qian Cheng Chun, and so on. EMD methods and Hilbert transform the treatment of boundary problems [J]. Chinese Science Bulletin, 2001, 46 (3) :257-263.

[18] P. Flandrin, G. Billing, P. Goncalves. Empirical Mode Decomposition as a Filter Bank [J].IEEE Signal Processing Letters, 2004, 11(2):112-114.

Ruiguo Sheng was born in Yantai, Shandong, China on March 21,1968 . He received the $\mathrm{PhD}$ degree in signal and information processing engineering in 2001 from the Harbin Industry Technology University. He works in Naval Aeronautical and Astronautical University, and is currently professor of Electronic Information Engineering Department. His research interests have included communication signal processing, wavelet theory and its application, etc..

Yang Zhang was born in Yantai, Shandong, China on March 1, 1983.He is the $\mathrm{PhD}$ Candidate in Electronic Information Engineering Department of Naval Aeronautical and Astronautical University from 2008. His research interests have included communication signal processing, nonlinear filter and chaos, etc.. 